\section{Conocimientos y prácticas sobre hidatidosis y triquinosis en usuarios y acompañantes del Hospital Comunitario de Salud Familiar El Carmen, Región del Biobío, Chile}

\author{
Raúl Lisboa-Navarro, Jorge González, Tania Junod, \\ Millaray Melín-Coloma y Carlos Landaeta-Aqueveque
}

Knowledge and practices regarding cystic echinococcosis and trichinellosis in users and companions of users of the Hospital Comunitario de Salud Familiar El Carmen, Biobio Region, Chile

We surveyed users of the Hospital Comunitario de Salud Familiar El Carmen and their companions to analyze knowledge and practices regarding cystic echinococcosis and trichinellosis. Most people recognized risky practices. Previous attendance at talks and working in agricultural-livestock enhanced the knowledge. Age, sex and formal education were not associated with the knowledge. Knowledge was not associated with practices.

Key words: Zoonosis, prevention, transmission, Trichinella, Echinococcus.

Palabras clave: Zoonosis, prevención, transmisión, Trichinella, Echinococcus.

\section{Introducción}

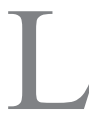

a hidatidosis es causada por céstodos del complejo Echinococcus granulosus sensu lato ${ }^{1}$. Los vermes adultos parasitan a los cánidos, mientras que en su estado larval, el quiste hidatídico, parasitan a diversos mamíferos, incluyendo humanos, los que se infectan mediante el consumo de huevos eliminados en las heces de los cánidos infectados. De este modo, la no desparasitación y la alimentación de perros con restos crudos de faena doméstica de animales favorecen la presentación de la hidatidosis humana ${ }^{2}$.

La triquinosis es causada por la ingestión de larvas de Trichinella spp. presentes en el músculo de hospederos infectados y causa una parasitosis intestinal y muscular. El cerdo es la fuente de infección más importante para el humano ${ }^{3}$. La crianza con prácticas poco higiénicas de cerdos, en presencia de ratas infectadas ${ }^{4} \mathrm{o}$ alimentados con restos de faena de otros $\operatorname{cerdos}^{3}$, junto con la ingestión de cecinas o carne mal cocida y sin la

Universidad de Concepción. Facultad de Ciencias Veterinarias (RLN, TJ, MMC, CLA).

Seremi de Salud Biobío. Delegación Provincial Ñuble (JG).

Los autores declaran que no existe conflicto de interés.

Financiamiento: El estudio fue financiado por la Delegación Provincial Ñuble de la Seremi de Salud Biobío.

Recibido: 3 de marzo de 2016 / Aceptado: 19 de julio de 2016

Correspondencia a:

Carlos Landaeta-Aqueveque

clandaeta@udec.cl inspección veterinaria previa ${ }^{5}$ son los principales factores de riesgo para la triquinosis.

Según datos disponibles en la Delegación Provincial Ñuble, Seremi de Salud Biobío, durante el quinquenio 2007-2011, la provincia de Ñuble fue la que alcanzó la mayor cantidad de casos de hidatidosis en la Región del Biobío, con 208 casos notificados. En esta provincia, la comuna de El Carmen registró la tercera tasa de incidencia más alta, con 28,8 casos por 100.000 habitantes, mucho mayor a la incidencia nacional $(1,4$ casos por 100.000 hab. en el año 2011) $)^{6}$. Por otro lado, en esta comuna también se han reportado tanto personas con triquinosis, como cerdos infectados con Trichinella spp. (18 personas en 2011 y ocho cerdos, desde 2010 a 2013).

La comuna de El Carmen, con una población proyectada al año 2013 de 11.284 habitantes, presenta factores de riesgo para ambas zoonosis, como pobreza económica $(24,8 \%)$, ruralidad $(58,6 \%)^{7}$ y carencia de plantas faenadoras de animales. Dado que los factores socioculturales pueden afectar la presencia de algunas zoonosis ${ }^{8-10}$, el diagnóstico del conocimiento y la conducta de la población es importante para el diseño de estrategias de prevención de estas enfermedades ${ }^{2,11-14}$

El Hospital Comunitario de Salud Familiar El Carmen es el principal centro de salud de la comuna, siendo un centro de baja complejidad (clasificación IV). En él se atiende casi la totalidad de la población rural de esta comuna, que es la población expuesta a ambas zoonosis parasitarias.

El objetivo de este estudio fue analizar el conocimiento y las prácticas de los usuarios del hospital y sus acompañantes, respecto de la hidatidosis $\mathrm{y}$ la triquinosis.

\section{Método}

Estudio de tipo transversal realizado sobre la base de encuestas en el período de enero y febrero de 2014. Se encuestaron 120 personas mayores de 18 años, quienes voluntariamente entregaron su consentimiento informado y estuvieron de acuerdo en responder la encuesta. El diseño de la encuesta se basó en estudios previos ${ }^{15}$ y fue validada previamente en la comuna de San Carlos, ubicada a $70 \mathrm{~km}$. de El Carmen, con características socioculturales similares a esta última. La encuesta se enfocó en seis aspectos: el conocimiento del concepto de zoonosis; conocimientos generales acerca de hidatidosis y triquinosis; conocimientos de los modos de transmisión de ambas enfermedades, (triquinosis: consumo de carne de cerdo o derivados mal cocidos; hidatidosis: consumo de huevos en alimentos contaminados o la interacción con perros infectados); conocimiento de los modos de prevención (triquinosis: crianza higiénica de cerdos y análisis triquinoscópico de la carne; hidatidosis: desparasitación de perros y alimentación de éstos sin restos crudos de faena); conductas asociadas con la infección (ambas enfermedades: faena domiciliaria; hidatidosis: perros sin desparasitación y alimentación de éstos con restos de faena; triquinosis: consumo de carne de cerdo sin análisis triquinoscópicos); y factores culturales y demográficos, como la edad, género, actividad laboral (agropecuaria, otra), educación (ninguna, básica, media, superior) y asistencia previa a charlas asociadas con estas enfermedades.

Para evaluar la asociación entre nivel de conocimientos, prácticas y factores que los afectan se utilizaron regresiones lineales, logísticas y pruebas no paramétricas.

Este trabajo fue certificado por el Comité de Ética de la Facultad de Ciencias Veterinarias de la Universidad de Concepción (Número de certificado: CBE352015). 
Resultados

El 49,2\% de los encuestados fueron mujeres; la edad promedio fue de 52 años; $23,8 \%$ finalizó la educación media; 50,8\% trabajaba en el ámbito agropecuario.

Respecto de los conocimientos, 27,5 y 7,5\% de los encuestados contestaron correctamente las preguntas sobre los modos de transmisión; y 40 y $9,2 \%$ contestaron correctamente las preguntas sobre prevención de triquinosis e hidatidosis, respectivamente. El nivel de conocimientos (porcentaje de preguntas correctas) fue de 65,6 y $16,6 \%$ para ambas enfermedades, respectivamente.

Respecto de las prácticas asociadas con triquinosis, $75 \%$ de los encuestados reconoció, al menos ocasionalmente, comer carne y derivados de cerdos faenados en el domicilio; y 43,3\%, al menos una vez, haber consumido dichos alimentos sin inspección veterinaria previa. En el caso de la hidatidosis, 59,2\% realizaba faenas domiciliarias de animales; $42,5 \%$ no había desparasitado sus perros en los últimos seis meses y $25 \%$ alimentaba a sus perros con vísceras crudas.

La asistencia previa a charlas y las actividades agropecuarias estuvieron asociadas a mayor conocimiento sobre la hidatidosis y triquinosis $(\mathrm{p}<0,001$ y $\mathrm{p}=0,022$, respectivamente). No hubo asociación entre la edad, género y nivel educacional con el conocimiento sobre las enfermedades, ni tampoco hubo asociación entre el nivel de conocimiento y las prácticas.

\section{Discusión}

Si bien el conocimiento del término zoonosis fue bajo, un alto porcentaje reconoció enfermedades zoonóticas, sugiriendo que las personas conocen la existencia de enfermedades transmisibles desde los animales. Estos resultados son similares a un estudio realizado en Pinto ${ }^{16}$, distante a unos $65 \mathrm{~km}$ de El Carmen, y en otros países sudamericanos ${ }^{14,17}$.

El conocimiento de las personas acerca de la triquinosis fue alto, probablemente por la condición de vivir en una zona endémica, factor asociado a un mayor conocimiento de las enfermedades ${ }^{14,18}$.

La proporción de personas que no realizaban el examen triquinoscópico de la carne sugiere un bajo impacto social de esta enfermedad, como se ha reportado en otros estudios ${ }^{19,20}$; o bien la falta de disponibilidad cercana del examen. Por su parte, la realización de faenas domiciliarias puede ser una práctica motivada, o bien obligada, por la carencia de plantas faenadoras en la comuna.

El conocimiento de las personas acerca de la hidatidosis fue bajo, al igual que lo reportado en otros estudios realizados en Sudamérica ${ }^{2,14,21}$, Chile central ${ }^{22}$ y hace tres décadas en la misma comuna ${ }^{23}$. Estudios previos han señalado la asociación entre un bajo nivel de conocimiento con la presencia de esta enfermedad ${ }^{11}$, lo que sugiere que la mantención de altas tasas de hidatidosis en El Carmen se puede deber a que el conocimiento de la enfermedad no ha mejorado en los últimos 30 años.

Aunque la desparasitación de los perros fue poco frecuente, sólo la cuarta parte de la población reconoció que los alimenta con vísceras crudas. Esta proporción no sólo fue menor que lo reportado en la Región del Maule ${ }^{22}$ y en otros países de Sudamérica ${ }^{13,24}$, sino que además fue significativamente menor que lo reportado previamente en la misma comuna $(100 \%)^{23}$.

En este estudio, no encontrar una asociación entre el nivel educacional y de conocimientos indica que la educación formal no fue suficiente en estos aspectos sanitarios. Esto sugiere la incorporación de estos conocimientos en los planes de educación formal en los colegios y liceos. No obstante, alternativas no formales o lúdicas de educación han demostrado tener buenos resultados y son muy adecuadas en zonas rurales ${ }^{22}$. La falta de asociación entre los conocimientos y las prácticas higiénicas, sumado a la carencia de plantas faenadoras y servicios veterinarios cercanos, sugiere que los esfuerzos deben enfocarse no sólo en medidas preventivas accesibles para las personas, (como evitar la alimentación de perros y cerdos con desechos crudos de faenas o la cocción adecuada de la carne de cerdo previo al consumo), sino que además en la necesidad de la desparasitación de perros y de visitas de plantas faenadoras móviles a localidades rurales distantes.

Agradecimientos: Al Hospital Comunitario de Salud Familiar de El Carmen por facilitar el lugar para realizar las encuestas.

\section{Resumen}

Introducción: La comuna de El Carmen, Región del Biobío, presenta factores de riesgo para la hidatidosis y la triquinosis, como un alto índice de pobreza, actividad principalmente agropecuaria y carencia de plantas faenadoras de animales. Método: Se analizó, a través de una encuesta a los usuarios y acompañantes del hospital de El Carmen, el conocimiento y las prácticas relacionadas con esas enfermedades. Resultados: La mayoría de los encuestados reconoció conductas de riesgo. La asistencia previa a charlas y el trabajo en actividades agropecuarias se asoció a mayor conocimiento de la hidatidosis y triquinosis, respectivamente. No hubo asociación entre edad, género o nivel educacional con el conocimiento de las enfermedades. Tampoco hubo asociación entre el conocimiento y las prácticas. Conclusiones: Los resultados sugieren la necesidad de mejoras educacionales, así como también intervenciones públicas, especialmente en sectores más lejanos.

\section{Referencias bibliográficas}

1.- Nakao M, Lavikainen A, Yanagida T, Ito A. Phylogenetic systematics of the genus Echinococcus (Cestoda: Taeniidae). Int J Parasitol 2013; 43: 1017-29.

2.- Moro P L, Cavero C A, Tambini M, Briceño Y, Jiménez R, Cabrera L. Prácticas, conocimientos y actitudes sobre la hidatidosis humana en poblaciones procedentes de zonas endémicas. Rev Gastroenterol Peru 2008; 28: 43-9.

3.- Pozio E. Searching for Trichinella: not all pigs are created equal. Trends Parasitol 2014; 30: 4-11.

4.- Takumi K, Teunis P, Fonville M, Vallee I, Boireau P, Nöckler K, et al. Transmission risk of human trichinellosis. Vet Parasitol 2009; 159: 324-7.

5.- Murrell K D, Pozio E. Worldwide occurrence and impact of human trichinellosis, 1986-2009. Emerg Infect Dis 2011; 17: 2194-202.

6.- Subsecretaría-de-Salud-Pública. Situación Epidemiológica de Zoonosis (Hantavirus, Triquinosis e Hidatidosis), Chile, 2014. 2016. Disponible en: http://www.seremidesaludbiobio.cl/epidemiologia/archivos/2014/seminario_ zoonosis/Vig\%20Epidem\%20Concepcion.pptm [Fecha acceso: 1 de julio de 2016].

7.- Guínez Cabezas D, Gaete Vásquez C. Diagnóstico El Carmen - Región del Biobío - MINSAL. 2013. Disponible en: http://www.seremidesaludbiobio. cl/epidemiologia/archivos/diagnosticos/2013/comunales/PROVINCIA_ NUBLE/elcarmen.pdf [Fecha acceso: 1 de julio de 2016]. 
8.- Vouga M, Greub G. Emerging bacterial pathogens: past and beyond. Clin Microbiol Infect 2016; 22: 12-21.

9.- Funk S, Salathé M, Jansen V A. Modelling the influence of human behaviour on the spread of infectious diseases: a review. J R Soc Interface 2010; 7 : 1247-56.

10.- Jones K E, Patel N G, Levy M A, Storeygard A, Balk D, Gittleman J L, et al. Global trends in emerging infectious diseases. Nature 2008; 451 : 990-3.

11.- Li D, Gao Q, Liu J, Feng Y, Ning W, Dong Y, et al. Knowledge, attitude, and practices (KAP) and risk factors analysis related to cystic echinococcosis among residents in Tibetan communities, Xiahe County, Gansu Province, China. Acta Trop 2015; 147: 17-22.

12.- Acosta-Jamett G, Weitzel T, Boufana B, Adones C, Bahamonde A, Abarca K, et al. Prevalence and risk factors for echinococcal infection in a rural area of northern Chile: a household-based cross-sectional study. PLoS Negl Trop Dis 2014; 8: e3090.

13.- Cabrera R, Talavera E, Trillo-Altamirano M P. Conocimientos, actitudes y prácticas de los matarifes acerca de la hidatidosis/equinococosis, en dos zonas urbanas del Departamento de Ica, Perú. An Fac Med Lima 2005; 66: 203-11.

14.- Molineri A I, Signorini M L, Tarabla H D. Conocimiento de las vías de transmisión de las zoonosis y de las especies afectadas entre los trabajadores rurales. Rev Argent Microbiol 2014; 46: 7-13.

15.- Isaza D M, Restrepo B N, Arboleda M, Casas E, Hinestroza H, Yurgaqui T. La leishmaniasis: conocimientos y prácticas en poblaciones de la costa del Pacífico de Colombia. Rev Panam Salud Publica 1999; 6: 177-84.
16.- Pino Bartolo F, Rojas P, Gädicke P. Evaluación del impacto de un programa de educación sanitaria para prevenir enfermedades zoonóticas. Theoria 2008; 17: 61-9.

17.- Cediel N, Villamil L C, Romero J, Renteria L, De Meneghi D. Establecimiento de prioridades en la vigilancia, la prevención y el control de las zoonosis en Bogotá, Colombia. Rev Panam Salud Publica 2013; 33: 31624.

18.- Gottstein B, Pozio E, Nöckler K. Epidemiology, diagnosis, treatment, and control of trichinellosis. Clin Microbiol Rev 2009; 22: 127-45.

19.- Conlan J V, Sripa B, Attwood S, Newton P N. A review of parasitic zoonoses in a changing Southeast Asia. Vet Parasitol 2011; 182: 22-40.

20.- Pozio E, Zarlenga D S. New pieces of the Trichinella puzzle. Int J Parasitol 2013; 43: 983-97.

21.- Reyes M M, Taramona C, Saire-Mendoza M, Guevara C, García H H. Disease awareness and knowledge in caregivers of children who had surgery for cystic hydatid disease in Lima, Peru. Trop Med Int Health 2010; 15: 1533-6.

22.- Apt W, Pérez C, Galdamez E, Campano S, Vega F, Vargas D, et al. Equinococosis/hidatidosis en la VII Región de Chile: diagnóstico e intervención educativa. Rev Panam Salud Publica 2000; 7: 8-16.

23.- Rubilar L, Zapata L, Moreno G, Cerda S. Prevalencia de Echinococcus granulosus y de otros cestodos del perro en la comuna de El Carmen, Nuble. Parasitol Dia 1985; 9: 55-7.

24.- González Peralta J, González G, Sbaffo A, Bessone A, Chassagnade M, Ugnia L, et al. Equinococosis canina en un sector del Departamento de Río Cuarto, Provincia de Córdoba, Argentina. Arch Med Vet 1998; 30: 157-63. 